NASA Contractor Report 181885

ICASE Report No. 89-48

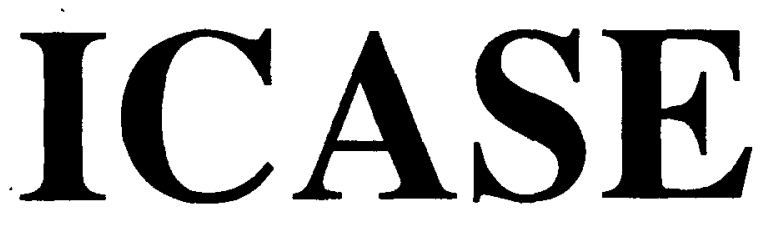

\title{
VARIATIONAL FORMULATION FOR A NONLINEAR ELLIPTIC EQUATION IN A THREE-DIMENSIONAL EXTERIOR DOMAIN
}

\section{Christine Bernardi \\ Vivette Girault \\ Laurence Halpern}

Contract No. NAS1-18605

July 1989

Institute for Computer Applications in Science and Engineering NASA Langley Research Center

Hampton, Virginia 23665-5225

Operated by the Universities Space Research Association

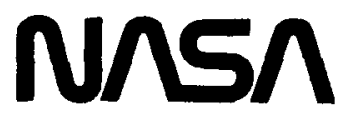

National Aeronaulics and

Space Administration 


\title{
VARIATIONAL FORMULATION FOR A NONLINEAR ELLIPTIC EQUATION IN A THREE-DIMENSIONAL EXTERIOR DOMAIN
}

\author{
Christine Bernardi* + \\ Vivette Girault $\ddagger$ \\ Laurence Halpern $\$$
}

\begin{abstract}
We obtain an existence result for a nonlinear second-order equation in an exterior domain of $I R^{3}$. The proof relies on a variational formulation in weighted Sobolev spaces.
\end{abstract}

* Research was supported in part for the first author by the National Aeronautics and Space Administration under NASA Contract No. NAS1-18605 while she was in residence at ICASE, NASA Langley Research Center, Hampton, VA 23665.

${ }^{\dagger}$ Analyse Numérique - \& Université Pierre Marie Curie - Tour 55-65, 5è étage 4 place Jussieu F-75252 Paris Cedex 05, FRANCE

${ }^{\ddagger}$ Analyse Numérique - \& Université Pierre Marie Curie - Tour 55-65, 5è étage 4 place Jussieu F-75252 Paris Cedex O5, FRANCE

Université de Paris, - Nord et Centre de Mathématiques Appliquées de l'École Polytechnique F-91128 Palaiseau Cedex, FRANCE 


\section{Introduction.}

In this paper, we are concerned with a nonlinear Laplace equation in an exterior 3-dimensional domain $\Omega$,

$$
-\Delta u+\mu|u|^{\sigma-1} u=f \quad \text { in } \Omega,
$$

provided with some Dirichlet conditions on the boundary of $\Omega$ and a condition of simple behaviour at infinity. Here, $\sigma$ is a real parameter, $3<\sigma<5$, and $\mu$ is equal to \pm 1 . We are presently unable to treat the two limit cases: $\sigma=3$, due to the lack of ellipticity of the linear term in our formulation, and $\sigma=5$ which is the critical exponent.

There is a wide literature devoted to equation (I.1), in the case $\mu=+1$ or in closely related cases. Without being exhaustive, we list here some outstanding references. The most general existence result is given in $[B]$, with no restriction on the behaviour of the solution and right-hand side at infinity. The existence of a ground-state solution (resp. of infinitely many radial solutions) is proved in [BeL1] (resp. [BeL2]) for special data. We also refer to the paper $[\mathrm{BaL}]$ and to the references therein. In the case of Neumann boundary conditions and zero right-hand side, the existence of ground-state solutions of a problem related to negative values of $\mu$ is established in [E].

We propose here a variational formulation of problem (I.1), which relies on weighted Sobolev spaces. None of the above references has used this approach. From the theoretical point of view, it has the advantage of leading to a new existence result when $\mu$ is equal to -1. And, from the practical point of view, it opens the perspective of numerical resolution.

We begin by writing a variational formulation of the associated linear equation

$$
-\Delta u=f \quad \text { in } \quad \Omega
$$

in appropriate weighted Sobolev spaces studied in $[\mathrm{BN}][\mathrm{G}][\mathrm{H}]$ among others: the weight is equal to $\left(1+r^{2}\right)^{\alpha}$, where $r$ denotes the distance to the origin, and $\alpha$ is a real parameter. That permits to prove that this problem is well-posed in such spaces for a range of values of $\alpha$. Finally, we derive an existence result for the full nonlinear equation by standard compactness arguments and sharp Sobolev embeddings.

An outline of the paper is as follows. In Section II, we recall the definition and we prove the basic properties of the weighted spaces we shall need. Section III is devoted to the analysis of the linear problem. The nonlinear equation is considered in Section IV.

\section{Properties of the weighted Sobolev spaces.}

In all that follows, the generic point of $\mathbb{R}^{3}$ is denoted by $\mathbf{x}=(x, y, z)$, and $r=r(\mathbf{x})$ is its distance to the origin. The notation $\mathrm{grad}$ represents the vector $\left(\frac{\partial}{\partial x}, \frac{\partial}{\partial y}, \frac{\partial}{\partial z}\right)$. For any triple $\lambda=\left(\lambda_{x}, \lambda_{y}, \lambda_{z}\right)$ of nonnegative integers, $|\lambda|$ stands for the sum $\lambda_{x}+\lambda_{y}+\lambda_{z}$ and, for any sufficiently smooth function $v$ defined on a part of $\mathbb{R}^{3}, D^{\lambda} v$ denotes the partial derivative $\frac{\partial^{\left.\lambda \lambda\right|_{v}}}{\partial x^{\lambda} \partial^{\lambda} y z^{\lambda z}}$. We shall always assume that $\Omega$ is an unbounded open set of $\mathbb{R}^{3}$, with boundary $\Gamma$ of class $\mathcal{C}^{\infty}$ and bounded. For any positive real number $R$, we denote by $\mathcal{O}_{R}$ the open ball centered at the origin with radius $R$. Finally, $\mathcal{O}$ stands for a fixed ball $\mathcal{O}_{R_{0}}$, where $R_{0}$ is large enough for $\mathcal{O}_{R_{0}}$ to contain $\Gamma$. 
Let $\alpha$ be a real parameter. For any real number $p, 1 \leq p \leq+\infty$, we define the space $L_{\alpha}^{p}(\Omega)$ by

$$
L_{\alpha}^{p}(\Omega)=\left\{v: \Omega \rightarrow \mathbb{R} \text { measurable; } v\left(1+r^{2}\right)^{\frac{\alpha}{2}} \in L^{p}(\Omega)\right\} \quad ;
$$

it is provided with the norm

$$
\|v\|_{0, p, \alpha}=\left(\int_{\Omega}|v|^{p}\left(1+r^{2}\right)^{\frac{p \alpha}{2}} d \mathbf{x}\right)^{\frac{1}{p}}
$$

Next, for any nonnegative integer $m$, we define the weighted Sobolev space $H_{\alpha}^{m}(\Omega)$ by

$$
H_{\alpha}^{m}(\Omega)=\left\{v: \Omega \rightarrow \mathbb{R} \text { measurable: } D^{\lambda} v\left(1+r^{2}\right)^{\frac{\alpha-m+|\lambda|}{2}} \in L^{2}(\Omega), 0 \leq|\lambda| \leq m\right\} ;
$$

it is provided with the norm

$$
\|v\|_{m, \alpha}=\left(\int_{\Omega_{0 \leq 1 \lambda \mid \leq m}}\left|D^{\lambda} v\right|^{2}\left(1+r^{2}\right)^{\alpha-m+|\lambda|} d \mathbf{x}\right)^{\frac{1}{2}}
$$

Equivalently, this scale of weighted Sobolev spaces can be defined by induction on $m$ via

$$
\begin{aligned}
& H_{\alpha}^{0}(\Omega)=L_{\alpha}^{2}(\Omega) \quad \text { and } \\
& H_{\alpha}^{m}(\Omega)=\left\{v \in H_{\alpha-1}^{m-1}(\Omega) ; \operatorname{grad} v \in\left(H_{\alpha}^{m-1}(\Omega)\right)^{3}\right\} \quad, \quad m \geq 1 .
\end{aligned}
$$

Finally, for any positive integer $m$, we denote by $\dot{H}_{\alpha}^{m}(\Omega)$ the closure in $H_{\alpha}^{m}(\Omega)$ of the space $\mathcal{D}(\Omega)$ of all indefinitely differentiable functions with compact support in $\Omega$, and by $H_{\alpha}^{-m}(\Omega)$ its dual space.

These spaces have been defined in a more general case and thoroughly studied $[\mathrm{BN}][\mathrm{H}]$, we only recall some basic properties we shall need.

1. For any nonnegative integer $m$, the space $\mathcal{D}(\bar{\Omega})$ of indefinitely differentiable functions with compact support in $\bar{\Omega}$ is dense in $H_{\alpha}^{m}(\Omega)$.

2. For any nonnegative integer $m$, the multiplication by a function of $\mathcal{D}(\bar{\Omega})$ is continuous from $H_{\alpha}^{m}(\Omega)$ into $H^{m}(\Omega)$. This allows to use the standard properties of the space $H^{m}(\Omega)$, especially the trace theorems.

3. For any nonnegative integer $m$ and for any real number $\beta$, the multiplication by $\left(1+r^{2}\right)^{\frac{0}{2}}$ is an isomorphism from $H_{\alpha}^{m}(\Omega)$ onto $H_{\alpha-\beta}^{m}(\Omega)$ and from $\dot{H}_{\alpha}^{m}(\Omega)$ onto $\dot{H}_{\alpha-\beta}^{m}(\Omega)$.

4. The mapping : $v \mapsto|v|_{1, \alpha}$ defined by

$$
|v|_{1, \alpha}=\left(\int_{\Omega}\left|\operatorname{grad}\left(v\left(1+r^{2}\right)^{\frac{\alpha}{2}}\right)\right|^{2} d \mathbf{x}\right)^{\frac{1}{2}}
$$

is a norm on $\dot{H}_{\alpha}^{1}(\Omega)$, equivalent to $\|\cdot\|_{1, \alpha}$.

5. The space $H_{\alpha}^{1}(\Omega)$ is contained in $L_{\alpha}^{6}(\Omega)$ with a continuous embedding.

First, we specify the constants involved in these last two properties. The following lemma is a generalization of Hardy's inequality [Nč, Chap. 6, Lemme 2.1]. 
Lemma 1. Any function $\chi$ in $\mathcal{D}\left(\overline{\mathbb{R}}_{+}\right)$satisfies

$$
\int_{0}^{+\infty} \chi(r)^{2} r^{2}\left(1+r^{2}\right)^{-1} d r \leq 4 \int_{0}^{+\infty} \chi^{\prime}(r)^{2} r^{2} d r
$$

Proof: A simple computation gives

$$
\begin{aligned}
0 & \leq \int_{0}^{+\infty}\left(\chi^{\prime}(r) r+\frac{1}{2} \chi(r) r^{2}\left(1+r^{2}\right)^{-1}\right)^{2} d r \\
\leq \int_{0}^{+\infty} \chi^{\prime}(r)^{2} r^{2} d r+ & \frac{1}{4} \int_{0}^{+\infty} \chi(r)^{2} r^{4}\left(1+r^{2}\right)^{-2} d r \\
& +\frac{1}{2} \int_{0}^{+\infty}\left(\chi^{2}\right)^{\prime}(r) r^{3}\left(1+r^{2}\right)^{-1} d r
\end{aligned}
$$

Since $r^{3}\left(1+r^{2}\right)^{-1}$ vanishes for $r=0$, we can integrate by parts:

$$
0 \leq \int_{0}^{+\infty} \chi^{\prime}(r)^{2} r^{2} d r-\frac{1}{2} \int_{0}^{+\infty} \chi(r)^{2} r^{2}\left(3+\frac{1}{2} r^{2}\right)\left(1+r^{2}\right)^{-2} d r,
$$

whence the lemma.

Lemma 2. For any function $v$ in $\dot{H}_{\alpha}^{1}(\Omega)$, the following estimates hold

$$
\|v\|_{0,6, \alpha} \leq \kappa|v|_{1, \alpha} \quad, \text { with } \kappa=\frac{1}{\sqrt{3}} \sqrt[3]{\frac{4}{\pi^{2}}}
$$

and

$$
\|v\|_{0,2, \alpha-1} \leq 2|v|_{1, \alpha}
$$

Proof: For any function $v$ in $\dot{H}_{\alpha}^{1}(\Omega)$, we denote by $\bar{v}$ its extension by 0 to $\mathbb{R}^{3}$ (note that $v$ vanishes on $\Gamma$, hence $\bar{v}$ belongs to $\left.H_{\text {loc }}^{1}\left(\mathbb{R}^{3}\right)\right)$. We set $w=\bar{v}\left(1+r^{2}\right)^{\frac{\alpha}{2}}$. To obtain the first bound, we recall the standard Sobolev inequality, valid for any function $\varphi$ in $\mathcal{D}\left(\mathbb{R}^{3}\right)$,

$$
\left(\int_{\mathbb{R}^{3}}|\varphi|^{6} d \mathbf{x}\right)^{\frac{1}{6}} \leq \kappa\left(\int_{\mathbb{R}^{3}}|\operatorname{grad} \varphi|^{2} d \mathbf{x}\right)^{\frac{1}{2}}
$$

where the optimal constant $\kappa$ is given in [T]. We apply this inequality, together with a density argument, to the function $w$. That gives

$$
\left\|v\left(1+r^{2}\right)^{\frac{\alpha}{2}}\right\|_{0,6,0} \leq \kappa\left(\int_{\Omega}\left|\operatorname{grad}\left(v\left(1+r^{2}\right)^{\frac{\alpha}{2}}\right)\right|^{2} d \mathbf{x}\right)^{\frac{1}{2}},
$$

which is exactly (II.8). Next, if $\mathcal{S}$ denotes the unit sphere of $\mathbb{R}^{3}$, we have

$$
\int_{\Omega} v^{2}\left(1+r^{2}\right)^{\alpha-1} d \mathrm{x}=\int_{S} \int_{0}^{+\infty} w^{2}\left(1+r^{2}\right)^{-1} r^{2} d r d \sigma
$$

and applying Lemma 1 together with a density argument gives

$$
\int_{\Omega} v^{2}\left(1+r^{2}\right)^{\alpha-1} d \mathbf{x} \leq 4 \int_{\mathcal{S}} \int_{0}^{+\infty}\left(\frac{\partial w}{\partial r}\right)^{2} r^{2} d r d \sigma \leq 4 \int_{\Omega}|\operatorname{grad} w|^{2} d \mathbf{x}
$$

whence (II.9).

Since we are concerned with second-order problems, we shall need some more properties of the space $H_{\alpha}^{1}(\Omega)$ that we are going to prove now. 
Lemma 3. For any real numbers $p$ and $\gamma$ satisfying

$$
2 \leq p \leq 6 \quad \text { and } \quad \gamma \leq \alpha+\frac{1}{2}-\frac{3}{p} \quad,
$$

the space $H_{\alpha}^{1}(\Omega)$ is contained in $L_{\gamma}^{p}(\Omega)$ with a continuous embedding.

Proof : From previous lines, we know that the space $H_{\alpha}^{1}(\Omega)$ is continuously embedded in both $L_{\alpha-1}^{2}(\Omega)$ and $L_{\alpha}^{6}(\Omega)$. Then, using Hölder's inequality, we write

$$
\begin{aligned}
\int_{\Omega}|v|^{p}\left(1+r^{2}\right)^{\frac{p \gamma}{2}} d \mathbf{x} & =\int_{\Omega}|v|^{3-\frac{p}{2}}\left(1+r^{2}\right)^{\frac{p \gamma}{2}-\frac{3 \alpha(p-2)}{4}}|v|^{3\left(\frac{p}{2}-1\right)}\left(1+r^{2}\right)^{\frac{3 \alpha(p-2)}{4}} d \mathbf{x} \\
& \leq\left(\int_{\Omega}|v|^{2}\left(1+r^{2}\right)^{\frac{2 p \gamma-3 \alpha(p-2)}{3-p}} d \mathbf{x}\right)^{\frac{8-p}{4}}\left(\int_{\Omega}|v|^{6}\left(1+r^{2}\right)^{3 \alpha} d \mathbf{x}\right)^{\frac{p-2}{4}} .
\end{aligned}
$$

We obtain

$$
\|v\|_{0, p, \gamma} \leq\|v\|_{0,2, \delta}^{\frac{3}{p}-\frac{1}{2}}\|v\|_{0,6, \alpha}^{\frac{3}{2}-\frac{3}{p}},
$$

with $\delta=\frac{2 p \gamma-3 \alpha(p-2)}{6-p}$. From (II.10), $\delta$ is less than $\alpha-1$ and the lemma follows.

Lemma 4. For any real number $\beta<\alpha-1$, the embedding of the space $H_{\alpha}^{1}(\Omega)$ into $L_{\beta}^{2}(\Omega)$ is compact.

Proof : Let $R$ be a real number larger than $R_{0}$. For any function $v$ in $H_{\alpha}^{1}(\Omega)$, we have

$$
\begin{aligned}
\int_{\Omega}|v|^{2}\left(1+r^{2}\right)^{\beta} d \mathbf{x} \leq & \int_{\Omega \cap \mathcal{O}_{R}}|v|^{2}\left(1+r^{2}\right)^{\beta} d \mathbf{x} \\
& \quad+\int_{\mathbb{R}^{3} \backslash \dot{\mathcal{O}}_{R}}|v|^{2}\left(1+r^{2}\right)^{\alpha-1}\left(1+R^{2}\right)^{\beta-(\alpha-1)} d \mathbf{x} \\
\leq & \max \left\{1,\left(1+R^{2}\right)^{\beta}\right\}\|v\|_{L^{2}\left(\mathcal{O}_{R}\right)}^{2}+\left(1+R^{2}\right)^{\beta-(\alpha-1)}\|v\|_{0,2, \alpha-1}^{2}
\end{aligned}
$$

Next, let $\left(w_{n}\right)_{n}$ be a sequence of $H_{\alpha}^{1}(\Omega)$ with $\left\|w_{n}\right\|_{1, \alpha} \leq 1$. Since this sequence is bounded, there exists a subsequence $\left(w_{n^{\prime}}\right)_{n^{\prime}}$ which converges to a function $w$ for the weak topology of $H_{\alpha}^{1}(\Omega)$. We apply the previous inequality to $w_{n^{\prime}}-w$ and use the fact that $\left\|w_{n^{\prime}}-w\right\|_{0,2, \alpha-1}$ is upbounded by $\left\|w_{n^{\prime}}-w\right\|_{1, \alpha}$, i.e., by 2 :

$$
\int_{\Omega}\left|w_{n^{\prime}}-w\right|^{2}\left(1+r^{2}\right)^{\beta} d \mathbf{x} \leq \max \left\{1,\left(1+R^{2}\right)^{\beta}\right\}\left\|w_{n^{\prime}}-w\right\|_{L^{2}\left(\mathcal{O}_{R}\right)}^{2}+2\left(1+R^{2}\right)^{\beta-(\alpha-1)} .
$$

For any positive real number $\varepsilon$, we choose $R$ large enough for $\left(1+R^{2}\right)^{\beta-(\alpha-1)}$ to be upbounded by $\frac{\varepsilon}{4}$. Next, it is clear that the sequence $\left(w_{n^{\prime} \mid \mathcal{O}_{R}}\right)_{n^{\prime}}$ is bounded in $H^{1}\left(\Omega \cap \mathcal{O}_{R}\right)$; since the embedding of $H^{1}\left(\Omega \cap \mathcal{O}_{R}\right)$ into $L^{2}\left(\Omega \cap \mathcal{O}_{R}\right)$ is compact, there exists a subsequence $\left(w_{n^{\prime \prime}}\right)_{n^{\prime \prime}}$ of $\left(w_{n^{\prime}}\right)_{n^{\prime}}$ which converges to $w$ in $L^{2}\left(\Omega \cap \mathcal{O}_{R}\right)$. For $n^{\prime \prime}$ large enough, the quantity $\left\|w_{n^{\prime \prime}}-w\right\|_{L^{2}\left(\mathcal{O}_{R}\right)}^{2}$ is upbounded by $\varepsilon / 2 \max \left\{1,\left(1+R^{2}\right)^{\beta}\right\}$, whence the lemma.

Corollary 1. For any real numbers $p$ and $\gamma$ satisfying

$$
2 \leq p<6 \quad \text { and } \quad \gamma<\alpha+\frac{1}{2}-\frac{3}{p} \quad,
$$


the embedding of the space $H_{\alpha}^{1}(\Omega)$ into $L_{\gamma}^{p}(\Omega)$ is compact.

Proof : With the same notation as in the previous proof, we apply (II.11) to the function $w_{n^{\prime \prime}}-w$, which gives

$$
\left\|w_{n^{\prime \prime}}-w\right\|_{0, \mathrm{p}, \gamma} \leq\left\|w_{n^{\prime \prime}}-w\right\|_{0,2,6}^{\frac{3}{p}-\frac{1}{2}}\left\|w_{n^{\prime \prime}}-w\right\|_{0,6, \alpha}^{\frac{3}{2}-\frac{3}{p}}
$$

From (II.12), it follows that $\delta$ is strictly less than $\alpha-1$ and the exponent $\frac{3}{p}-\frac{1}{2}$ of the norm $\|\cdot\|_{0,2, \delta}$ is positive, whence the corollary.

Remark 1: The purpose of the weight $\left(1+r^{2}\right)^{\alpha}$ is to enforce a certain behaviour at infinity. For instance, suppose a function $v$ behaves like $\left(1+r^{2}\right)^{\beta}$ at infinity, i.e., suppose it satisfies for $R$ large enough

$$
\forall \mathbf{x} \in \mathbb{R}^{3} \backslash \overline{\mathcal{O}}_{R}, \quad|v(\mathbf{x})| \leq c\left(1+r^{2}\right)^{\beta} \quad \text { and } \quad|\operatorname{grad} v(\mathbf{x})| \leq c\left(1+r^{2}\right)^{\beta-1 / 2}
$$

then it belongs to $H_{\alpha}^{1}(\Omega)$ if $\beta$ is strictly smaller than $-\frac{\alpha}{2}-\frac{1}{4}$. Hence, if $\alpha$ is greater than $-\frac{1}{2}$, a function $v$ which satisfies (II.13) and belongs to $H_{\alpha}^{1}(\Omega)$, tends to 0 at infinity.

\section{The linear problem.}

Let $\alpha$ be a fixed parameter. Assume that a distribution $f$ is given in $H_{\alpha}^{-1}(\Omega)$. Our purpose is to search for solutions of the problem

$$
\begin{aligned}
-\Delta u=f & \text { in } \Omega \\
u=0 & \text { on } \Gamma
\end{aligned}
$$

Clearly, this problem admits the following equivalent variational formulation: Find $u$ in $\dot{H}_{\alpha}^{1}(\Omega)$ such that

$$
\forall v \in \dot{H}_{\alpha}^{1}(\Omega), \quad a_{\alpha}(u, v)=\langle f, v\rangle_{\alpha} \quad,
$$

where the bilinear form $a_{\alpha}(.,$.$) is defined by$

$$
\forall u \in H_{\alpha}^{1}(\Omega), \forall v \in H_{\alpha}^{1}(\Omega), \quad a_{\alpha}(u, v)=\int_{\Omega} \operatorname{grad} u \cdot \operatorname{grad}\left(v\left(1+r^{2}\right)^{\alpha}\right) d \mathbf{x}
$$

and $\langle., .\rangle_{\alpha}$ stands for the duality pairing between $\dot{H}_{\alpha}^{1}(\Omega)$ and $H_{\alpha}^{-1}(\Omega)$. Note that this formulation generalizes the result of [G].

Remark 2: Let us for a while consider the problem with inhomogeneous boundary conditions

$$
\begin{aligned}
-\Delta u=f & \text { in } \Omega, \\
u=g & \text { on } \Gamma,
\end{aligned}
$$

where $g$ is a given function in $H^{1 / 2}(\Gamma)$. Due to the trace theorem, there exists a function $u_{b}$ in $H^{1}(\Omega \cap \mathcal{O})$ such that its trace on $\Gamma$ is $g$ and which vanishes on $\partial \mathcal{O}$. Extending this function by 0 to $\mathbb{R}^{3}$, we obtain a function $\bar{u}_{b}$ of $H_{\alpha}^{1}(\Omega)$. Next, a function $u$ is a solution of (III.4) if and only if the function $u^{*}=u-\vec{u}_{b}$ is a solution of problem (III.1) with $\mathrm{f}$ replaced by $f^{\bullet}=f+\Delta \bar{u}_{b}$. 
Remark 3: As noted in Remark 1, there are cases where the fact that the solution belongs to $H_{\alpha}^{1}(\Omega)$ forces it to tend to 0 at infinity. However, we can be interested in seeking a solution of problem (III.1) or (III.4) which satisfies

$$
\lim _{r(\mathbf{x}) \rightarrow+\infty} u(\mathbf{x})=u_{\infty}
$$

where $u_{\infty}$ is a given constant. Then, it suffices to search $u-u_{\infty}$ as a solution of problem (III. 4 ) with $g$ replaced by $-u_{\infty}$ or $g-u_{\infty}$.

In order to analyse problem (III.1), we need some properties of the form $a_{\alpha}(.,$.$) .$

Proposition 1. Let $\alpha$ be any real parameter. The form $a_{\alpha}(.,$.$) is continuous on H_{\alpha}^{1}(\Omega) \times$ $H_{\alpha}^{1}(\Omega)$ with its norm upbounded by $\sqrt{1+4 \alpha^{2}}$.

Proof: Let $u$ and $v$ be in $H_{\alpha}^{1}(\Omega)$. we compute

$$
\begin{aligned}
a_{\alpha}(u, v)=\int_{\Omega}(\operatorname{grad} u) & \cdot(\operatorname{grad} v)\left(1+r^{2}\right)^{\alpha} d \mathbf{x} \\
& +2 \alpha \int_{\Omega}(\operatorname{grad} u) \cdot(v \mathbf{x})\left(1+r^{2}\right)^{\alpha-1} d \mathbf{x}
\end{aligned}
$$

This implies

$$
\begin{aligned}
a_{\alpha}(u, v) & \leq\|\operatorname{grad} u\|_{0,2, \alpha}\left(\|\operatorname{grad} v\|_{0,2, \alpha}+2|\alpha|\|v\|_{0,2, \alpha-1}\right) \\
& \leq \sqrt{1+4 \alpha^{2}}\|u\|_{1, \alpha}\|v\|_{1, \alpha},
\end{aligned}
$$

whence the proposition.

Let us recall that the norm $|\cdot|_{1, \alpha}$ defined in (II.6) is equivalent to $\|\cdot\|_{1, \alpha}$ on $\dot{H}_{\alpha}^{1}(\Omega)$.

Proposition 2. Let $\alpha$ be a real parameter, $-\frac{1}{2}<\alpha<\frac{1}{2}$. The form $a_{\alpha}(.,$.$) satisfies the$ property of ellipticity

$$
\forall u \in \dot{H}_{\alpha}^{1}(\Omega), \quad a_{\alpha}(u, u) \geq\left(1-4 \alpha^{2}\right)|u|_{1, \alpha}^{2}
$$

Proof: Let $u$ be a function of $\dot{H}_{\alpha}^{1}(\Omega)$, we set $w=u\left(1+r^{2}\right)^{\frac{\alpha}{2}}$. It has already been recalled that $w$ belongs to $\dot{H}_{0}^{1}(\Omega)$. We now compute

$$
\begin{aligned}
a_{\alpha}(u, u) & =\int_{\Omega} \operatorname{grad}\left(w\left(1+r^{2}\right)^{-\frac{\alpha}{2}}\right) \cdot \operatorname{grad}\left(w\left(1+r^{2}\right)^{\frac{q}{2}}\right) d \mathbf{x} \\
& =\int_{\Omega}|\operatorname{grad} w|^{2} d \mathbf{x}-\alpha^{2} \int_{\Omega} w^{2} r^{2}\left(1+r^{2}\right)^{-2} d \mathbf{x}
\end{aligned}
$$

Next, we extend $w$ by 0 on $\mathbb{R}^{3} \backslash \bar{\Omega}$ and denote the extension by $\bar{w}$. If $\mathcal{S}$ denotes the unit sphere of $\mathbb{R}^{3}$, we have

$$
\int_{\Omega} w^{2} r^{2}\left(1+r^{2}\right)^{-2} d \mathbf{x}=\int_{\mathcal{S}} \int_{0}^{+\infty} \bar{w}^{2} r^{2}\left(1+r^{2}\right)^{-2} r^{2} d r d \sigma
$$


and applying Lemma 1 together with a density argument gives

$$
\int_{\Omega} w^{2} r^{2}\left(1+r^{2}\right)^{-2} d \mathbf{x} \leq 4 \int_{\mathcal{S}} \int_{0}^{+\infty}\left(\frac{\partial \bar{w}}{\partial r}\right)^{2} r^{2} d r d \sigma \leq 4 \int_{\Omega}|\operatorname{grad} w|^{2} d \mathbf{x}
$$

Finally, we obtain

$$
a_{\alpha}(u, u) \geq\left(1-4 \alpha^{2}\right) \int_{\Omega}|\operatorname{grad} w|^{2} d \mathbf{x}
$$

But $\int_{\Omega}|\operatorname{grad} w|^{2} d \mathbf{x}$ is precisely equal to $|u|_{1, \alpha}^{2}$.

Remark 4: Let us prove, by a simple counter-example, that the range of values of $\alpha$ for which the form $a_{\alpha}(.,$.$) is elliptic cannot be extended. Let us assume for a while that \Omega$ is the whole space $\mathbb{R}^{3}$. As already mentioned, the function $u_{\beta}: \mathbf{x} \mapsto\left(1+r(\mathbf{x})^{2}\right)^{\beta}$ belongs to $H_{\alpha}^{1}\left(\mathbb{R}^{3}\right)$ if and only if $\beta$ is $<-\frac{\alpha}{2}-\frac{1}{4}$. A simple computation gives

$$
a_{\alpha}\left(u_{\beta}, u_{\beta}\right)=c(\alpha, \beta)\left|u_{\beta}\right|_{1, \alpha}^{2} \quad, \text { with } c(\alpha, \beta)= \begin{cases}0 & \text { if } \beta(\alpha+\beta)=0 \\ \frac{1}{1+\frac{\alpha^{2}}{4 \beta(\alpha+\rho)}} & \text { otherwise }\end{cases}
$$

If $\alpha$ is $<-\frac{1}{2}$ (resp. $>\frac{1}{2}$ ), choosing $\beta=0$ (resp. $\beta=-\alpha$ ) gives $c(\alpha, \beta)=0$. If $\alpha$ is equal to $-\frac{1}{2}$ (resp. $\frac{1}{2}$ ), the constant $c(\alpha, \beta)$ tends to 0 when $\beta$ tends to 0 (resp. $-\frac{1}{2}$ ). When $\alpha$ is $<-\frac{1}{2}$ or $>\frac{1}{2}$, the same counter-example proves that the inf-sup conditions

$$
\begin{aligned}
& \inf _{u \in H_{\alpha}^{1}(\Omega)} \sup _{v \in H_{\alpha}^{1}(\Omega)} \frac{a_{\alpha}(u, v)}{|u|_{1, \alpha}|v|_{1, \alpha}}>0, \\
& \inf _{v \in H_{\alpha}^{1}(\Omega)} \sup _{u \in H_{\alpha}^{1}(\Omega)} \frac{a_{\alpha}(u, v)}{|u|_{1, \alpha}|v|_{1, \alpha}}>0
\end{aligned}
$$

do not hold simultaneously, and this simultaneity is a necessary condition for problem (III.2) to be well-posed.

The following theorem is a straightforward consequence of the Lax-Milgram Lemma and Propositions 1 and 2.

Theorem 1. Let $\alpha$ be a real parameter, $-\frac{1}{2}<\alpha<\frac{1}{2}$. For every $f$ in $H_{\alpha}^{-1}(\Omega)$, problem (III.2) has a unique solution $u$ in $\dot{H}_{\alpha}^{1}(\Omega)$. This solution satisfies

$$
|u|_{1, \alpha} \leq \frac{1}{1-4 \alpha^{2}} \sup _{v \in A_{\alpha}^{1}(\Omega)} \frac{\langle f, v\rangle_{\alpha}}{|v|_{1, \alpha}}
$$

Remark 5: Assume that $f$ belongs to $H_{\alpha}^{-1}(\Omega)$ with $-\frac{1}{2}<\alpha<\frac{1}{2}$. Then, the solution $u$ of problem (III.2) belongs to $\dot{H}_{\beta}^{1}$ for any real number $\beta<\alpha$. It coincides with the unique solution of problem (III.2) with $\alpha$ replaced by $\beta$ if $\beta$ is $>-\frac{1}{2}$. If $\beta$ is less than $-\frac{1}{2}$, it is still a solution of this problem. That amounts to saying that we have written an infinity of variational formulations (III.2) corresponding to the same problem (III.1).

Remark 6: One could think of applying similar techniques to study the linear fourth-order equation

$$
\begin{array}{cc}
\Delta^{2} u=f & \text { in } \Omega, \\
u=\frac{\partial u}{\partial n}=0 & \text { on } \Gamma,
\end{array}
$$


where $\frac{\partial}{\partial n}$ denotes the normal derivative on $\Gamma$. This equation can be written in a variational form: Find $u$ in $\dot{H}_{\alpha}^{2}(\Omega)$ such that

$$
\forall v \in \dot{H}_{\alpha}^{2}(\Omega), \quad b_{\alpha}(u, v)=\left\langle\langle f, v\rangle_{\alpha} \quad\right.
$$

where the bilinear form $b_{\alpha}(.,$.$) is defined by$

$$
\forall u \in H_{\alpha}^{2}(\Omega), \forall v \in H_{\alpha}^{2}(\Omega), \quad b_{\alpha}(u, v)=\int_{\Omega} \Delta u \Delta\left(v\left(1+r^{2}\right)^{\alpha}\right) d \mathbf{x}
$$

and $\left\langle\langle., .\rangle_{\alpha}\right.$ stands for the duality pairing between $\dot{H}_{\alpha}^{2}(\Omega)$ and $H_{\alpha}^{-2}(\Omega)$. However, if $\Omega$ is the whole space $\mathbb{R}^{3}$, it can easily be checked that the function $u_{-\alpha}=\left(1+r^{2}\right)^{-\alpha}$ satisfies $b_{\alpha}\left(u_{-\alpha}, u_{-\alpha}\right)=0$ and belongs to $\dot{H}_{\alpha}^{2}(\Omega)$ for any $\alpha>-\frac{1}{2}$. Hence, problem (III.8) cannot be well-posed in this space. It must be set instead in a quotient space.

\section{The nonlinear equation.}

We now turn to problem (I.1). In order to be a little less restrictive, we introduce a function $\psi$ defined on $\Omega \times \mathbb{R}$, satisfying $\psi(., 0)=0$ and we assume that it satisfies the following inequality for some nonnegative real numbers $\sigma$ and $\tau$ :

$$
\forall(\xi, \eta) \in \mathbb{R}^{2}, \quad|\psi(., \xi)-\psi(., \eta)| \leq \tau\left(|\xi|^{\sigma-1}+|\eta|^{\sigma-1}\right)|\xi-\eta| \quad \text { a.e. in } \Omega \text {. }
$$

Next, for a real parameter $\alpha$ and a given distribution $f$ in $H_{\alpha}^{-1}(\Omega)$, we consider the problem

$$
\begin{aligned}
-\Delta u+\psi(., u) & =f & & \text { in } \Omega \\
u & =0 & & \text { on } \Gamma \quad
\end{aligned} .
$$

In order to write a variational formulation for this problem, we first determine the values of $\sigma$ for which the function: $u \mapsto \psi(., u)$ maps $\dot{H}_{\alpha}^{1}(\Omega)$ into its dual.

Lemma 5. Let $\alpha$ be a nonnegative real parameter. For any real number $\sigma$ satisfying

$$
1+\frac{4}{2 \alpha+1} \leq \sigma \leq 5
$$

the function $\Psi: u \mapsto \psi(., u)$ maps the space $\dot{H}_{\alpha}^{1}(\Omega)$ into $H_{\alpha}^{-1}(\Omega)$. Moreover, for any functions $u$ and $v$ in $H_{\alpha}^{1}(\Omega)$, the following estimate holds

$$
\left|\int_{\Omega} \Psi(u) v\left(1+r^{2}\right)^{\alpha} d \mathbf{x}\right| \leq c\|u\|_{1, \alpha}^{\sigma}\|v\|_{1, \alpha} .
$$

Proof: Let $p$ be a real number, $2 \leq p \leq 6$, and define $p^{*}$ by $\frac{1}{p}+\frac{1}{p^{*}}=1$. For any functions $u$ and $v$ in $H_{\alpha}^{1}(\Omega)$, we have from $(T V .1)$

$$
\begin{aligned}
\left|\int_{\Omega} \Psi(u) v\left(1+r^{2}\right)^{\alpha} d \mathbf{x}\right| & \leq \tau \int_{\Omega}|u|^{\sigma}|v|\left(1+r^{2}\right)^{\alpha} d \mathbf{x} \\
& \leq \tau \int_{\Omega}|u|^{\sigma}\left(1+r^{2}\right)^{\frac{1}{2}\left(\alpha+\frac{3}{2}-\frac{3}{p^{*}}\right)}|v|\left(1+r^{2}\right)^{\frac{1}{2}\left(\alpha+\frac{1}{2}-\frac{3}{p}\right)} d \mathbf{x} \\
& \leq \tau\left(\int_{\Omega}|u|^{p^{*} \sigma}\left(1+r^{2}\right)^{\frac{p^{*}}{2}\left(\alpha+\frac{3}{2}-\frac{3}{p^{*}}\right)} d \mathbf{x}\right)^{\frac{1}{p^{*}}} \\
& \left(\int_{\Omega}|v|^{p}\left(1+r^{2}\right)^{\frac{p}{2}\left(\alpha+\frac{1}{2}-\frac{3}{p}\right)} d \mathbf{x}\right)^{\frac{1}{p}}
\end{aligned}
$$


This finally gives

$$
\left|\int_{\Omega} \Psi(u) v\left(1+r^{2}\right)^{\alpha} d \mathbf{x}\right| \leq \tau\|u\|_{0, p^{*} \sigma, \frac{\alpha+\delta / 2-3 / p^{*}}{\sigma}}^{\sigma}\|v\|_{0, p, \alpha+\frac{1}{2}-\frac{3}{p}} .
$$

Next, it follows from Lemma 3 that $\|v\|_{0, p, \alpha+\frac{1}{2}-\frac{3}{p}}$ is upbounded by $c\|v\|_{1, \alpha}$ and that $\|u\|_{0, p^{*} \sigma, \frac{\alpha+s / 2-3 / p^{*}}{\sigma}}$ is bounded by $c\|u\|_{1, \alpha}$ if

$$
2 \leq p^{*} \sigma \leq 6 \quad \text { and } \quad \alpha+\frac{5}{2}-\frac{3}{p^{*}} \leq \sigma\left(\alpha+\frac{1}{2}-\frac{3}{p^{*} \sigma}\right)
$$

Since $p^{*}$ must be chosen between $\frac{6}{5}$ and 2 , this condition is equivalent to (IV.3).

Lemma 6. Let $\alpha$ be a nonnegative real parameter. For any real number $\sigma$ satisfying (IV.3) the function $\Psi: u \mapsto \psi(., u)$ is continuous from the space $\dot{H}_{\alpha}^{1}(\Omega)$ into $H_{\alpha}^{-1}(\Omega)$. Moreover, for any functions $u, w$ and $v$ in $H_{\alpha}^{1}(\Omega)$, the following estimate holds

$$
\left|\int_{\Omega}(\Psi(u)-\Psi(w)) v\left(1+r^{2}\right)^{\alpha} d \mathbf{x}\right| \leq c\left(\|u\|_{1, \alpha}^{\sigma-1}+\|w\|_{1, \alpha}^{\sigma-1}\right)\|u-w\|_{1, \alpha}\|v\|_{1, \alpha}
$$

Proof: With the same notation as in the previous proof and in the same way, we compute from (IV.1)

$$
\begin{aligned}
& \left|\int_{\Omega}(\Psi(u)-\Psi(w)) v\left(1+r^{2}\right)^{\alpha} d \mathbf{x}\right| \\
& \leq \tau\left\{\left(\int_{\Omega}|u|^{p^{*}(\sigma-1)}|u-w|^{p^{*}}\left(1+r^{2}\right)^{\frac{p^{*}}{2}\left(\alpha+\frac{3}{2}-\frac{3}{\left.p^{*}\right)}\right.} d \mathbf{x}\right)^{\frac{1}{p^{+}}}\right. \\
& \left.+\left(\int_{\Omega}|w|^{p^{*}(\sigma-1)}|u-w|^{p^{*}}\left(1+r^{2}\right)^{\frac{p^{*}}{2}\left(\alpha+\frac{3}{2}-\frac{3}{p^{*}}\right)} d \mathbf{x}\right)^{\frac{1}{p^{*}}}\right\} \\
& \left(\int_{\Omega}|v|^{p}\left(1+r^{2}\right)^{\frac{p}{2}\left(\alpha+\frac{1}{2}-\frac{3}{p}\right)} d \mathbf{x}\right)^{\frac{1}{p}} \\
& \leq \tau\left\{\left(\int_{\Omega}|u|^{p^{*}(\sigma-1)}|u-w|^{p^{*}}\left(1+r^{2}\right)^{\frac{p^{*}}{2}\left(\alpha+\frac{3}{2}-\frac{3}{\left.p^{*}\right)}\right.} d \mathbf{x}\right)^{\frac{1}{p^{*}}}\right. \\
& \left.+\left(\int_{\Omega}|w|^{p^{*}(\sigma-1)}|u-w|^{p^{*}}\left(1+r^{2}\right)^{\frac{p^{*}}{2}\left(\alpha+\frac{3}{2}-\frac{3}{p^{*}}\right)} d \mathbf{x}\right)^{\frac{1}{p^{*}}}\right\}\|v\|_{0, p, \alpha+\frac{1}{2}-\frac{3}{p}}
\end{aligned}
$$

We use once more Hölder's inequality to estimate the first term

$$
\begin{aligned}
\int_{\Omega}|u|^{p^{*}(\sigma-1)}|u-w|^{p^{*}}\left(1+r^{2}\right)^{\frac{p^{*}}{2}\left(\alpha+\frac{3}{2}-\frac{3}{\left.p^{*}\right)} d \mathbf{x}\right.} & \\
\leq & \left(\int_{\Omega}|u|^{p^{*} \sigma}\left(1+r^{2}\right)^{\frac{p^{*}}{2}\left(\alpha+\frac{5}{2}-\frac{3}{p^{*}}\right)} d \mathbf{x}\right)^{\frac{\sigma-1}{\sigma}} \\
& \left(\int_{\Omega}|u-w|^{p^{*} \sigma}\left(1+r^{2}\right)^{\frac{p^{*}}{2}\left(\alpha+\frac{3}{2}-\frac{3}{p^{*}}\right)} d \mathbf{x}\right)^{\frac{1}{\sigma}}
\end{aligned}
$$


The second term is treated in a similar way. Finally we obtain

$$
\left|\int_{\Omega}(\Psi(u)-\Psi(w)) v\left(1+r^{2}\right)^{\alpha} d \mathbf{x}\right| \leq \tau\left(\|u\|_{\left.0, p^{*} \sigma, \frac{\alpha+s / 2-3 / p^{*}}{\sigma-1}+\|w\|_{\left.0, p^{*} \sigma, \frac{\alpha+\beta / 2-3 / p^{*}}{\sigma}\right)}^{\sigma-1}\right)}^{\|u-w\|_{0, p^{*} \sigma, \frac{\alpha+3 / 2-3 / p^{*}}{\sigma}\|v\|_{0, p, \alpha+\frac{1}{2}-\frac{3}{p}} .} .}\right.
$$

Then, we conclude as previously.

We will also need the following corollary, which is an immediate consequence of equation (IV.7).

Corollary 2. Let $\alpha$ be a positive real parameter. For any real number $\sigma$ satisfying

$$
1+\frac{4}{2 \alpha+1}<\sigma<5,
$$

there exist real numbers $q$ and $\delta$ verifying

$$
2 \leq q<6 \quad \text { and } \quad \delta<\alpha+\frac{1}{2}-\frac{3}{q}
$$

such that, for any functions $u, v$ and $w$ in $H_{\alpha}^{1}(\Omega)$, the following estimate holds

$$
\left|\int_{\Omega}(\Psi(u)-\Psi(w)) v\left(1+r^{2}\right)^{\alpha} d \mathbf{x}\right| \leq c\left(\|u\|_{1, \alpha}^{\sigma-1}+\|w\|_{1, \alpha}^{\sigma-1}\right)\|u-w\|_{0, q, \delta}\|v\|_{1, \alpha}
$$

We now see that problem (IV.2) admits the variational formulation: Find $u$ in $\dot{H}_{\alpha}^{1}(\Omega)$ such that

$$
\forall v \in \dot{H}_{\alpha}^{1}(\Omega), \quad a_{\alpha}(u, v)+\int_{\Omega} \psi(\mathbf{x}, u) v\left(1+r^{2}\right)^{\alpha} d \mathbf{x}=\langle f, v\rangle_{\alpha}
$$

We are in a position to state the main results about equation (IV.2). It is the object of the two following theorems, that we prove simultaneously. Let us introduce the constant

$$
\rho_{\alpha}=\left(1-4 \alpha^{2}\right)^{\frac{\sigma}{\sigma-1}} \tau^{-\frac{1}{\sigma-1}} 2^{-\frac{\delta-\sigma}{2(\sigma-1)}} \kappa^{-\frac{3}{2}}(\sigma-1) \sigma^{-\frac{\sigma}{\sigma-1}},
$$

where $\kappa$ is defined in (II.8).

Theorem 2. Let $\alpha$ be a real parameter, $0 \leq \alpha<\frac{1}{2}$. Assume that the function $\psi$ satisfies the assumption (IV.1) for real numbers $\sigma$ verifying (IV.8) and $\tau$. Then, for every $f$ in $H_{\alpha}^{-1}(\Omega)$ satisfying

$$
\sup _{v \in H_{\alpha}^{1}(\Omega)} \frac{\langle f, v\rangle_{\alpha}}{|v|_{1, \alpha}}<\rho_{\alpha}
$$

problem (IV.11) has a solution $u$ in $\dot{H}_{\alpha}^{1}(\Omega)$.

Theorem 3. Let $\alpha$ be a real parameter, $0<\alpha<\frac{1}{2}$. Assume that the function $\psi$ satisfies the assumption (IV.1) for real numbers $\sigma$ verifying (IV.8) and $\tau$, and moreover that

$$
\forall v \in \dot{H}_{\alpha}^{1}(\Omega), \quad \int_{\Omega} \dot{\psi}(\mathbf{x}, v) v\left(1+r^{2}\right)^{\alpha} d \mathbf{x} \geq 0
$$


Then, for every $f$ in $H_{\alpha}^{-1}(\Omega)$, problem $(I V .11)$ has a solution $u$ in $\dot{H}_{\alpha}^{1}(\Omega)$.

Proof: Due to the density of $\mathcal{D}(\Omega)$ in $\dot{H}_{\alpha}^{1}(\Omega)$, the space $\dot{H}_{\alpha}^{1}(\Omega)$ is separable. Let $\left(v_{m}\right)_{m \in \mathbb{N}}$ be a total family in $\dot{H}_{\alpha}^{1}(\Omega)$. Next, for any nonnegative integer $m$, we denote by $V_{m}$, the subspace spanned by $\left\{v_{0}, \ldots, v_{m}\right\}$, and we consider the problem $(P)_{m}:$ Find $u_{m}$ in $V_{m}$ such that

$$
\forall v_{m} \in V_{m}, \quad a_{\alpha}\left(u_{m}, v_{m}\right)+\int_{\Omega} \psi\left(\mathbf{x}, u_{m}\right) v_{m}\left(1+r^{2}\right)^{\alpha} d \mathbf{x}=\left\langle f, v_{m}\right\rangle_{\alpha} .
$$

In order to prove that this problem has at least one solution, we define an operator $\Phi_{m}$ from $\dot{H}_{\alpha}^{1}(\Omega)$ into $V_{m}$ : for any $v$ in $\dot{H}_{\alpha}^{1}(\Omega), \Phi_{m}(v)$ belongs to $V_{m}$ and is such that

$$
\forall v_{m} \in V_{m}, \quad\left(\Phi_{m}(v), v_{m}\right)_{\alpha}=a_{\alpha}\left(v, v_{m}\right)+\int_{\Omega} \psi(\mathbf{x}, v) v_{m}\left(1+r^{2}\right)^{\alpha} d \mathbf{x}-\left\langle f, v_{m}\right\rangle_{\alpha} \quad,
$$

where $(., .)_{\alpha}$ denotes the scalar product of $\dot{H}_{\alpha}^{1}(\Omega)$ associated with the norm $|.|_{1, \alpha}$. Hence, a function $u_{m}$ of $V_{m}$ will be a solution of problem $(P)_{m}$ if and only if it satisfies $\Phi_{m}\left(u_{m}\right)=0$. For $v_{m}$ in $V_{m}$, let us compute $\left(\Phi_{m}\left(v_{m}\right), v_{m}\right)_{\alpha}$. If the assumptions of Theorem 2 are satisfied, it follows from Proposition 2 and (IV.5) that, for a suitable value of $p$,

$$
\begin{gathered}
\left(\Phi_{m}\left(v_{m}\right), v_{m}\right)_{\alpha} \geq\left(1-4 \alpha^{2}\right)\left|v_{m}\right|_{1, \alpha}^{2}-\tau\left\|v_{m}\right\|_{0, p^{*} \sigma,}^{\sigma} \frac{\frac{\alpha+3 / 2-3 / p^{*}}{\sigma}}{\sigma}\left\|v_{m}\right\|_{0, p, \alpha+\frac{1}{2}-\frac{3}{p}} \\
-\|f\|_{H_{\alpha}^{-1}}\left|v_{m}\right|_{1, \alpha},
\end{gathered}
$$

where $\|\cdot\|_{H_{\alpha}^{-1}}$ is the dual norm of $|\cdot|_{1, \alpha}$. Applying (II.11) and Lemma 2 gives

$$
\begin{gathered}
\left(\Phi_{m}\left(v_{m}\right), v_{m}\right)_{\alpha} \geq\left(\left(1-4 \alpha^{2}\right)\left|v_{m}\right|_{1, \alpha}-\tau 2^{\sigma\left(\frac{3}{p^{-\sigma}}-\frac{1}{2}\right)} \kappa^{\sigma\left(\frac{3}{2}-\frac{3}{p^{-\alpha}}\right)} 2^{\frac{3}{p}-\frac{1}{2}} \kappa^{\frac{3}{2}-\frac{3}{p}}\left|v_{m}\right|_{1, \alpha}^{\sigma}\right. \\
\left.-\|f\|_{H_{\alpha}^{-1}}\right)\left|v_{m}\right|_{1, \alpha},
\end{gathered}
$$

whence finally

$$
\left(\Phi_{m}\left(v_{m}\right), v_{m}\right)_{\alpha} \geq\left(\left(1-4 \alpha^{2}\right)\left|v_{m}\right|_{1, \alpha}-\tau 2^{\frac{s-\sigma}{2}} \kappa \frac{3(\sigma-1)}{2}\left|v_{m}\right|_{1, \alpha}^{\sigma}-\|f\|_{H_{\alpha}^{-1}}\right)\left|v_{m}\right|_{1, \alpha} .
$$

If (IV.13) is satisfied with the value of $\rho_{\alpha}$ defined in (IV.12), the quantity $\left(\Phi_{m}\left(v_{m}\right) \cdot v_{m}\right)_{\alpha}$ is nonnegative when

$$
\left|v_{m}\right|_{1, \alpha}=\lambda=\left(\frac{1-4 \alpha^{2}}{\sigma \tau 2^{\frac{3-\sigma}{2}} \kappa^{\frac{3(\sigma-1)}{2}}}\right)^{\frac{1}{\sigma-1}}
$$

If the assumptions of Theorem 3 are satisfied, due to ( $I V .14)$, we simply have

$$
\left(\Phi_{m}\left(v_{m}\right), v_{m}\right)_{\alpha} \geq\left(\left(1-4 \alpha^{2}\right)\left|v_{m}\right|_{1, \alpha}-\|f\|_{H_{\alpha}^{-1}}\right)\left|v_{m}\right|_{1, \alpha} \quad,
$$

so that $\left(\Phi_{m}\left(v_{m}\right), v_{m}\right)_{\alpha}$ is nonnegative when, for instance,

$$
\left|v_{m}\right|_{1, \alpha}=\lambda=\frac{2\|f\|_{H_{a}^{-1}}}{1-4 \alpha^{2}}
$$


In both cases, applying a corollary of the Brouwer's fixed-point theorem [GR, Chapter IV, Corollary 1.1], we deduce that $\Phi_{m}$ has a zero $v_{m}$ in $V_{m}$, the norm of which is bounded by $\lambda$, i.e., independently of $m$.

Thus, we have built a bounded sequence $\left(u_{m}\right)_{m \in \mathbb{N}}$ of $\dot{H}_{\alpha}^{1}(\Omega)$. From Corollary 1, we know that there exists a subsequence $\left(u_{m^{\prime}}\right)_{m^{\prime}}$ which converges to $u$ weakly in $\dot{H}_{\alpha}^{1}(\Omega)$ and strongly in $L_{\delta}^{q}(\Omega)$, for the real numbers $q$ and $\delta$ of Corollary 2. Applying these convergences in problem $(P)_{m^{\prime}}$ and using Corollary 2 , we deduce that $u$ is a solution of problem (IV.11).

Remark 7: For a given real number $\sigma, 3<\sigma<5$, choosing the smallest parameter $\alpha$ such that (IV.8) holds implies that $\rho_{\alpha}$ must satisfy

$$
\rho_{\alpha}<(8(\sigma-3))^{\frac{\sigma}{\sigma-1}}(\sigma-1)^{-\frac{\sigma+1}{\sigma-1}} \tau^{-\frac{1}{\sigma-1}} 2^{-\frac{3-\sigma}{2(\sigma-1)}} \kappa^{-\frac{3}{2}} \sigma^{-\frac{\sigma}{\sigma-1}} .
$$

This bound tends to 0 when $\sigma$ tends to 3 and to $\frac{2}{5} \pi \sqrt[4]{\frac{27}{5 \tau}} \simeq 1.915 \tau^{-\frac{1}{4}}$ when $\sigma$ tends to 5 .

Remark 8: In the previous theorems, the parameter $\alpha$ is always larger than $-\frac{1}{2}$. Hence, it follows from Remark 1 that, if the solution of problem (IV.11) satisfies (II.13), it tends to 0 at infinity.

The last result about equation (IV.1) concerns the uniqueness of the solution.

Proposition 3. Let $B$ denote the ball

$$
B=\left\{v \in \dot{H}_{\alpha}^{1}(\Omega) ;|v|_{1, \alpha}<\kappa^{-\frac{3}{2}}\left(\frac{1-4 \alpha^{2}}{\tau 2^{\frac{7-\alpha}{2}}}\right)^{\frac{1}{\sigma-1}}\right\} .
$$

Problem (IV.11) has at most one solution in the ball $B$.

Proof: Let $u$ and $w$ be two solutions of (IV.11). We have

$$
\forall v \in \dot{H}_{\alpha}^{1}(\Omega), \quad a_{\alpha}(u-w, v)=-\int_{\Omega}(\Psi(u)-\Psi(w)) v\left(1+r^{2}\right)^{\alpha} d \mathbf{x}
$$

Choosing $v=u-w$ and applying Proposition 2, (IV.7), (II.11) and Lemma 2 give as previously

$$
\left(1-4 \alpha^{2}\right)|u-w|_{1, \alpha}^{2} \leq \tau 2^{\frac{5-\sigma}{2}} \kappa^{\frac{3(\sigma-1)}{2}}\left(|u|_{1, \alpha}^{\sigma-1}+|w|_{1, \alpha}^{\sigma-1}\right)|u-w|_{1, \alpha}^{2} \quad,
$$

whence the result.

Remark 9: A particular case of equation (IV.2) is given by

$$
\begin{aligned}
-\Delta u+\mu|u|^{\sigma-1} u & =f & \text { in } \Omega, \\
u & =0 & \text { on } \Gamma,
\end{aligned},
$$

where $\mu$ is a real number. Theorems 2 and 3 state that this problem has at least a variational solution for $3<\sigma<5$ respectively when $\mu$ is negative and $f$ is small enough, or when $\mu$ is nonnegative. Proposition 3 provides a result of local uniqueness. 


\section{References}

[BaL] A. BAHRI \& P.-L. LIONS - Remarques sur la thèorie variationnelle des points critiques et applications, Comptes-Rendus de l'Académie des Sciences 301 (1985), pp. 145-147.

[BN] J. BARROS NETO - Inhomogeneous Boundary Value Problems in a Half-Space, Ann. Sc. Norm. Sup. Pisa 19 (1965), pp. 331-365.

[BeL1] H. BERESTYCKI \& P.-L. LIONS - Nonlinear scalar field equations, I. Existence of a ground state, Arch. Rat. Mech. Mat. 82 (1983), pp. 313-346.

[BeL2] H. BERESTYCKI \& P.-L. LIONS - Nonlinear scalar field equations, II. Existence of infinitely many solutions, Arch. Rat. Mech. Mat. 82 (1983), pp. 347-375.

[B] B. BREZIS - Semilinear equations in $\mathbb{R}^{N}$ without condition at infinity, Appl. Math. Optim. 12 (1984), pp. 271-282.

[E] M. ESTEBAN - Rupture de symétrie pour des problèmes de Neumann sur-linéaires dans des ouverts extérieurs, Comptes-Rendus de l'Académie des Sciences (1989) (to appear).

[GR] V. GIRAULT \& P.-A. RAVIART - Finite Element Methods for Navier-Stokes Equations. Theory and Algorithms, Springer-Verlag (1986).

[G] J. GIROIRE - Étude de quelques problèmes aux limites extérieurs et résolution par équations intégrales, Thèse, Université Pierre et Marie Curie (1987).

[H] B. HANOUZET - Espaces de Sobolev avec poids, application au problème de Dirichlet dans un demi-espace, Rend. del Sem. della Univ. di Padova XLVI (1971), pp. 227-272.

[Nč] J. NEČAS - Les Méthodes Directes en Théorie des Équations Elliptiques, Masson (1967).

[T] G. TALENTI - Best constants in Sobolev inequality, Ann. Mat. Pura Appl. 110 (1976), pp. 353-372. 


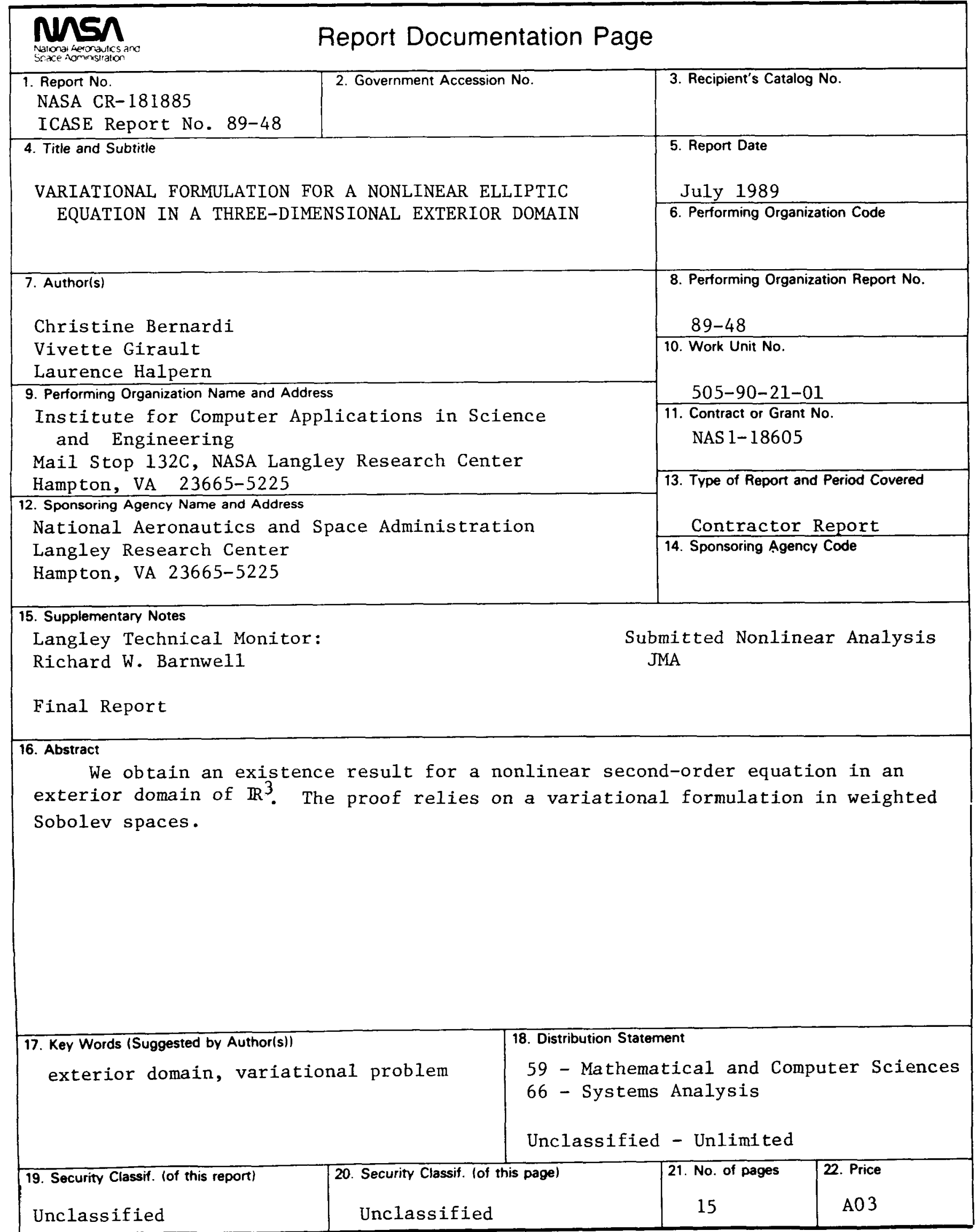

